\title{
NECESSARY AND SUFFICIENT CONDITIONS FOR EVENTUALLY VANISHING OSCILLATORY SOLUTIONS OF FUNCTIONAL EQUATIONS WITH SMALL DELAYS
}

\author{
BHAGAT SINGH \\ Department of Mathematics \\ University of Wisconsin Center \\ 705 Viebahn Street \\ Manitowoc, Wisconsin 54220 U.S.A.
}

(Received March 28, 1978 and in Revised form June 30, 1978)

ABSTRACT. Necessary and sufficient conditions are found for all oscillatory solutions of the equation

$$
\left(r_{n-1}(t)\left(r_{n-2}(t)\left(---\left(r_{2}(t)\left(r_{1}(t) y^{\prime}(t)\right)^{\prime}\right)^{\prime}\right)^{\prime}---\right)\right)^{\prime}+a(t) h(y(g(t)))=b(t)
$$

to approach zero. Sufficient conditions are also given to ensure that all solutions of this equation are unbounded.

KEY WORDS AND PHRASES. Oscillatory, Nonoscillatory, Delay, Functional. AMS (MOS) SUBJECT CLASSIFICATION (1970) CODES.

1. INTRODUCTION.

Recently, T. Kusano and H. Onose [6] studied the equation $\left(r_{n-1}(t)\left(r_{n-2}(t)\left(---\left(r_{2}(t)\left(r_{1}(t) y^{\prime}(t)\right)^{\prime}\right)^{\prime}---\right)^{\prime}\right)^{\prime}\right)^{\prime}+a(t) h(y(g(t)))=b(t)$ and found sufficient conditions which force all bounded nonoscillatory solutions of (1) to approach zero when $a(t)$ is oscillatory. For positive $a(t)$, the same 
conditions ensured that all nonoscillatory solutions of (1) approached zero. Kartsatos [4, Theo. 1] also found sufficient criteria for all bounded nonoscillatory solutions of (1) to, asymptotically, vanish generalizing results of this author and Dahiya [7, Theo. 1]. In fact, since the work of Hammett [3] such asymptotic results about the nonoscillatory solutions of ordinary and retarded differential equations have been obtained by many authors such as Kartsatos [4], Kusano and Onose [5, 6], this author and Dahiya [7], this author [9, 10, 11] and many others. A fairly exhaustive list of references on oscillation can be found in Graef [2]. Most of these results relate to nonoscillation properties of solutions. Very little has been said about the asymptotic nature of the corresponding oscillatory solutions of these equations. This author's work $[8,9,12]$ is devoted to this type of study about the oscillatory solutions of such equations.

our purpose in this paper is to further the study initiated by Kusano and onose [6] and find necessary and sufficient conditions to ensure that all oscillatory solutions of equation (1) tend to zero as $t \rightarrow \infty$. In the last section, we give sufficient conditions which cause all solutions of (1) to be unbounded. Chen [1] studied a similar problem but our results are different and more extensive.

In what follows, we shall restrict our study to those solutions of which can be continuously extended on some positive half line, say for $t \geq t_{0}>0$. We shall, therefore, assume the point $t_{0}$ fixed for the rest of this paper. The term "solution" applies only to continuously extendable solutions on $\mathrm{R}^{+}=\left[t_{0}, \infty\right)$.

\section{DEFINITIONS AND ASSUMPTIONS.}

The following conditions hold for the rest of this paper:

(i) $a(t), b(t), g(t), r_{1}(t),---, r_{n-1}(t)$ are real valued and continuous 
on $[t, \infty), h \varepsilon C(-\infty, \infty) ;$
(ii) $g(t) \leq t, g(t) \rightarrow \infty$ as $t \rightarrow \infty ;$
(iii) $t h(t)>0, t \neq 0 ;$
(iv) there exists a number $m$ such that

$$
\frac{h(t)}{t} \leq m
$$

(v) $r_{i}(t) \geq \alpha>0, i=1,2, \ldots, n-1$; for large $t$ on $\mathrm{R}^{+}$.

A solution is said to be oscillatory if it has arbitrarily large zeros, otherwise it is said to be nonoscillatory. To further shorten notations we designate:

$$
\begin{aligned}
& z_{1} y(t) \equiv\left(r_{1}(t) y^{\prime}(t)\right)^{\prime}, z_{2} y(t) \equiv\left(r_{2}(t)\left(r_{1}(t) y^{\prime}(t)\right)^{\prime}\right)^{\prime}, \cdots-\cdots, \\
& z_{i} y(t) \equiv\left(r_{i}(t)\left(r_{i-1}(t)\left(---\left(r_{2}(t)\left(r_{1}(t) y^{\prime}(t)\right)^{\prime}\right)^{\prime}---\right)^{\prime}\right)^{\prime}\right)^{\prime}, \\
& i=1,2,-\cdots, n-1 .
\end{aligned}
$$

\section{MAIN RESULTS.}

LEMMA (3.1). Suppose $\int^{\infty}|\mathrm{a}(t)| d t<\infty$ and $\int^{\infty}|\mathrm{b}(t)| d t<\infty$. Let $y(t)$ be a bounded oscillatory solution of (1). Then

$$
\frac{r_{i+1}\left(z_{i Y}(t)\right)}{t^{n-i-2}} \rightarrow 0
$$

as $t \rightarrow \infty, i=1,2, \cdots, n-2$.

PROOF, Since $y(t)$ is oscillatory, $z_{i} y(t)$ is oscillatory for $i=1$, $2,-\cdots, n-1$. Let $\varepsilon>0$ be arbitrary and let $t_{1}>t_{0}$ be so large that $z_{n-2} Y\left(t_{1}\right)=0$,

$$
\operatorname{mm}_{t_{1}}^{\infty}|a(t)| d t<\varepsilon / 2
$$

and

$$
\int_{t_{1}}^{\infty}|b(t)| a t \leq \varepsilon / 2,
$$

where $|y(g(t))| \leq M$ for $t \geq t_{1}$. 
Integrating equation (1) we have

$$
\left|r_{n-1}(t) z_{n-2} y(t)\right| \leq \operatorname{mM}_{t_{1}}^{t}|a(x)| d x+\int_{t_{1}}^{t}|b(x)| d x \leq \varepsilon
$$

Thus $z_{n-2} y(t) \rightarrow 0$ as $t \rightarrow \infty$.

Let now $t_{2}>t_{1}$ be a zero of $z_{n-3} y(t)$ so that $\left|r_{n-1}(t) z_{n-2} y(t)\right| \leq \varepsilon$ for $t \geq t_{2}$. Now

$$
r_{n-2}(t) z_{n-3} y(t)=\int_{t}^{t} z_{n-2} y(s) d s
$$

which readily gives

$$
\frac{\left|r_{n-2}(t) z_{n-3} y(t)\right|}{t} \leq \frac{\varepsilon\left(t-t_{2}\right)}{t} \leq \varepsilon .
$$

Proceeding this way, the proof is completed.

LEMMA (3.2). Suppose $\int^{\infty}|\mathrm{a}(t)| d t<\infty, \int^{\infty}|\mathrm{b}(t)| d t<\infty$ and

$$
\frac{1}{r_{1}(t)}=O\left(\frac{1}{t^{n-\beta}}\right)
$$

for some $B \in[0,1)$. Then oscillatory solutions of (1) are bounded.

PROOF. Let $T>t_{0}$ be large enough so that for $t \geq T, g(t)>t_{0}$.

Integrating (1) (for $t \geq T$ ) over $\left[t_{0}, t\right]$ we have

$$
\begin{aligned}
z_{n-2}(t) y(t)=\frac{1}{r_{n-1}(t)} r_{n-1}\left(t_{0}\right) z_{n-2} y\left(t_{0}\right) & -\frac{1}{r_{n-1}(t)} \int_{t_{0}}^{t} a(x) h(y(g(x))) d x \\
& +\frac{1}{r_{n-1}(t)} \int_{t_{0}}^{t} b(x) d x .
\end{aligned}
$$

On repeated integration (10) yields

$$
\begin{aligned}
& r_{1}(t) y^{\prime}(t)=r_{1}\left(t_{0}\right) y^{\prime}\left(t_{0}\right)+r_{2}\left(t_{0}\right) z_{1} y\left(t_{0}\right) \int_{t_{0}}^{t} 1 / r_{2}(x) d x \\
& +r_{3}\left(t_{0}\right) z_{2}\left(y\left(t_{0}\right)\right) \int_{t_{0}}^{t} 1 / r_{2}\left(x_{2}\right) \int_{t_{0}}^{x_{2}} 1 / x_{3}(x) d x d x_{2}+\cdots \\
& +\cdots+r_{n-1}\left(t_{0}\right) z_{n-2} y\left(t_{0}\right) \int_{t_{0}}^{t_{1}} 1 / r_{2}\left(x_{2}\right) \int_{t_{0}}^{x_{2}} 1 / r_{3}--\int_{t_{0}}^{x_{n-2}} 1 / r_{n-1}(x) d x-\cdots d x_{2} \\
& -\int_{t_{0}}^{t} 1 / r_{2}\left(x_{2}\right) \int_{t_{0}}^{x_{2}} 1 / r_{3}\left(x_{3}\right) \int_{t_{0}}^{x_{3}}---\int_{t_{0}}^{x_{n-2}} \frac{1}{r_{n-1}\left(x_{n-1}\right)} \int_{t_{0}}^{x_{n-1}} a(x) h(y(g(x))) d x-\cdots d x_{2}
\end{aligned}
$$




$$
\begin{aligned}
& +\int_{t_{0}}^{t} 1 / r_{2}\left(x_{2}\right) \int_{t_{0}}^{x_{2}} 1 / r_{3}\left(x_{3}\right) \int_{t_{0}}^{x_{3}} 1 / r_{4}\left(x_{4}\right) \cdots \int_{t_{0}}^{x_{n-2}} \frac{1}{r_{n-1}\left(x_{n-1}\right)} \\
& \int_{t_{0}}^{x_{n-1} b(x) d x-\cdots d x_{2}}
\end{aligned}
$$

Dividing (11) by $r_{1}(t)$ and integrating between $t_{0}$ and $g(t)$ we have $y(g(t))=y\left(g\left(t_{0}\right)\right)+r_{1}\left(t_{0}\right) y^{\prime}\left(t_{0}\right) \int_{t_{0}}^{g(t)} 1 / r_{1}(x) d x+r_{2}\left(t_{0}\right) z_{1} y\left(t_{0}\right)$

$$
\int_{t_{0}}^{g(t)} 1 / r_{1}\left(x_{1}\right) \int_{t_{0}}^{x_{1}}{ }_{1 / r_{2}}(x) d x d x_{1}
$$

$+r_{3}\left(t_{0}\right) z_{2} y\left(t_{0}\right) \int_{t_{0}}^{g(t)} 1 / r_{1}\left(x_{1}\right) \int_{t_{0}}^{x_{1}} 1 / r_{2}\left(x_{2}\right) \int_{t_{0}}^{x_{2}} 1 / r_{3}(x) d x d x_{2} d x_{1}$

$+\cdots+r_{n-1}\left(t_{0}\right) z_{n-2} y\left(t_{0}\right) \int_{t_{0}}^{g(t)} 1 / r_{1}\left(x_{1}\right) \int_{t_{0}}^{x_{1}} 1 / r_{2}\left(x_{2}\right) \ldots$

$$
\int_{t_{0}}^{x_{n-2}} \frac{1}{r_{n-1}(x)} d x d x_{n-2}-\cdots d x_{1}
$$

$$
\begin{aligned}
& -\int_{t_{0}}^{g(t)} 1 / \tilde{x}_{1}\left(x_{1}\right) \int_{t_{0}}^{x_{1}} 1 / r_{2}\left(x_{2}\right) \int_{t_{0}}^{x_{2}} 1 / x_{3}\left(x_{3}\right) \cdots+\int_{t_{0}}^{x_{n-2}} 1 / r_{n-1}\left(x_{0}\right) \\
& \int_{t_{0}}^{x_{0}} a(x) h(y(g(x))) d x d x_{0} \ldots d x_{1}
\end{aligned}
$$$$
+\int_{t_{0}}^{g(t)} 1 / r_{1}\left(x_{1}\right) \int_{t_{0}}^{x_{1}} 1 / r_{2}\left(x_{2}\right) \int_{t_{0}}^{x_{2}} 1 / r_{3}\left(x_{3}\right) \cdots \int_{t_{0}}^{x_{n-2}} 1 / r_{n-1}\left(x_{n-1}\right)
$$

$$
\int_{t_{0}}^{x_{n-1}} b(x) d x d x_{n-1} d x_{n-2} d x_{n-3}--d x_{1}
$$

Since each $1 / r_{i}(t) \leq \frac{1}{\alpha}, i=2,3, \ldots n-1$ and $g(t) \leq t$ we have from above

$$
\begin{aligned}
|y(g(t))| & \leq\left|y\left(g\left(t_{0}\right)\right)\right|+\left|r_{1}\left(t_{0}\right) y^{\prime}\left(t_{0}\right)\right| \int_{t_{0}}^{t} 1 / r_{1}(x) d x \\
& +\frac{1}{\alpha}\left|r_{2}\left(t_{0}\right) z_{1} y\left(t_{0}\right)\right| \int_{t_{0}}^{t}\left(x-t_{0}\right) / r_{1}(x) d x
\end{aligned}
$$




$$
\begin{aligned}
& +\frac{1}{2 ! \alpha^{2}}\left|r_{3}\left(t_{0}\right) z_{2} y\left(t_{0}\right)\right| \int_{t_{0}}^{t}\left(x-t_{0}\right)^{2} / r_{1}(x) d x \\
& + \\
& +\frac{1}{(n-2) ! \alpha^{n-2}}\left|r_{n-1}\left(t_{0}\right) z_{n-2} y\left(t_{0}\right)\right| \int_{t_{0}}^{t}\left(x-t_{0}\right)^{n-2} / x_{1}(x) d x \\
& +\frac{1}{(n-2) ! \alpha^{n-2}} \int_{t_{0}}^{t_{1}(x)} \int_{t_{0}}^{r_{1}}(x-s)^{n-2}|a(s)||h(y(g(s)))| d s d x \\
& +\frac{1}{(n-2) !} \frac{1}{\alpha^{n-2}} \int_{t_{0}}^{t} \frac{1}{r_{1}(x)} \int_{t_{0}}^{x}(x-s)^{n-2}|b(s)| d s d x .
\end{aligned}
$$

Due to conditions on $r_{1}(t)$ we find that each term on the right hand side of (13) except possibly last two are bounded, and since $h(t) / t \leq m$, there exist constants $\mathrm{K}_{1}, \mathrm{~K}_{2}$ and $\mathrm{K}_{3}$ such that

$$
\begin{aligned}
|y(g(t))| & \leq k_{1}+k_{2} \int_{t_{0}}^{t} \int_{t_{0}}^{x} \frac{(x-s)^{n-2}}{x^{n-\beta}}|a(s)||y(g(s))| d s d x \\
& +k_{3} \int_{t_{0}}^{t} \int_{t_{0}}^{x} \frac{(x-s)^{n-2}}{x^{n-\beta}}|b(s)| d s d x .
\end{aligned}
$$

Rearranging constants still further we get

$$
\begin{aligned}
|y(g(t))| & \leq k_{1}+k_{4} \int_{t_{0}}^{t} \int_{0}^{x} \frac{|a(s)||y(g(s))| d s d x}{x^{2-\beta}} \\
& +k_{5} \int_{t_{0}}^{t} \int_{t_{0}}^{x} \frac{|b(s)| d s d x}{x^{2-\beta}} \\
& =k_{1}+k_{4} \int_{t_{0}}^{t}\left(\int_{s}^{t} \frac{1}{x^{2-\beta}} d x\right)|a(s)||y(g(s))| d s \\
& +k_{5} \int_{t_{0}}^{t}\left(\int_{s}^{t} \frac{1}{x^{2-\beta}} d x\right)|b(s)| d s
\end{aligned}
$$

by change of order of integration. Now 


$$
\int_{s}^{t} \frac{1}{x^{2-\beta}} d x \leq c
$$

for some constant $C>0$ since $0 \leq \beta<1$. Thus the last term in (14) is bounded, since $\int^{\infty}|\mathrm{b}(\mathrm{s})| \mathrm{ds}<\infty$.

From (14) and (15), there exists a positive constant $K_{6}$ such that $|y(g(t))| \leq K_{6}+k_{5} c \int_{t_{0}}^{t}|a(s)||y(g(s))| d s$.

By Gronwall's inequality $\mathrm{y}(\mathrm{g}(\mathrm{t}))$ is bounded and the proof is complete. THEOREM (3.1). Subject to the conditions of Lemma 3.2 all oscillatory solutions of equation (1) approach zero.

PROOF. Suppose to the contrary that some oscillatory solution $y(t)$ of (1) is such that

$$
\lim _{t \rightarrow \infty}|y(t)|>3 d>0
$$

for some number $d$. Let $T>t_{0}$ be large enough so that for $t \geq T$ we have (from lemma 3.1)

$$
\frac{r_{i+1}(t)\left(z_{i} Y(t)\right)}{i ! \alpha^{i} t^{n-i-2}}<\frac{d}{n}, i=1,2, \ldots, n-2
$$

It follows from (4) that

$$
\frac{r_{1}(t) y^{\prime}(t)}{t^{n-2}} \rightarrow 0 \text { as } t \rightarrow \infty \text {. }
$$

Let now $T_{0}>T$ be a zero of $Y(t)$ so that for $t \geq T_{0^{\prime}}$ (17) and (18) imply

$$
\frac{r_{i+1}(t) z_{i}(y(t))}{i ! \alpha^{i} t^{n-i-2}}<\frac{d}{n}, i=1,2,-\cdots, n-2,
$$

and

$$
\frac{r_{1}(t) y^{\prime}(t)}{t^{n-2}}<\frac{d}{n} .
$$

Integrating ( 1 ) between $\left[\mathrm{T}_{0}, t\right]$ we have 


$$
\begin{aligned}
& y(t)=r_{1}\left(T_{0}\right) y^{\prime}\left(T_{0}\right) \int_{T_{0}}^{t} 1 / r_{1}(x) d x+r_{2}\left(T_{0}\right) z_{1}\left(T_{0}\right) \int_{T_{0}}^{x_{1}} 1 / r_{2}\left(x_{1}\right) \int_{T_{0}}^{x_{1}} 1 / r_{2}(x) d x d x_{1} \\
& +r_{3}\left(T_{0}\right) z_{2} Y\left(T_{0}\right) \int_{T_{0}}^{t} 1 / r_{1}\left(x_{1}\right) \int_{T_{0}}^{x_{1}} 1 / x_{2}\left(x_{2}\right) \int_{T_{0}}^{x_{2}} 1 / r_{3}(x) d x d x_{2} d x_{1} \\
& +\cdots+r_{n-1}\left(T_{0}\right) z_{n-2} Y\left(T_{0}\right) \int_{T_{0}}^{t} 1 / r_{1}\left(x_{1}\right) \int_{T_{0}}^{x_{1}} 1 / r_{2}\left(x_{2}\right) \cdots \int_{T_{0}}^{x_{n-2}} 1 / r_{n-1} \\
& (x) d x d x_{n-2}--d x_{1} \\
& -\int_{T_{0}}^{t} 1 / x_{1}\left(x_{1}\right) \int_{T_{0}}^{x_{1}} 1 / r_{2}\left(x_{2}\right) \cdots-\int_{T_{0}}^{x_{n-2}} 1 / r_{n-1}\left(x_{n-1}\right) \int_{T_{0}}^{x_{n-1}} a(x) h(y(g(x))) d x d x_{n-1} \\
& --d x_{1} \\
& +\int_{T_{0}}^{t} 1 / x_{1}\left(x_{1}\right) \int_{T_{0}}^{x_{1}} 1 / x_{2}\left(x_{2}\right) \int_{T_{0}}^{x_{2}} 1 / x_{3}\left(x_{3}\right) \cdots \int_{T_{0}}^{x_{n-1}} b(x) d x d x_{n-1} \cdots d x_{1} .
\end{aligned}
$$

Since $y(t)$ is bounded, let $|y(g(t))| \leq c_{1}$ for some positive constant $\mathrm{C}_{1}$. From (20) we have

$$
\begin{aligned}
|y(t)| & \leq\left|r_{1}\left(T_{0}\right) y^{\prime}\left(T_{0}\right)\right| \int_{T_{0}}^{t} 1 / r_{1}(x) d x+\frac{1}{\alpha}\left|r_{2}\left(T_{0}\right) z_{1} y\left(T_{0}\right)\right| \int_{T_{0}}^{t} \frac{\left(x-T_{0}\right)}{r_{1}(x)} d x \\
& +\frac{1}{2 ! \alpha^{2}}\left|r_{3}\left(T_{0}\right) z_{2} y\left(T_{0}\right)\right| \int_{T_{0}}^{t}\left(x-T_{0}\right)^{2} / r_{1}(x) d x \\
& +- \\
& +\frac{1}{(n-2) ! \alpha^{n-2}} \int_{T_{0}}^{t}\left(x-T_{0}\right)^{n-2} / r_{1}(x) d x \\
& +\frac{c_{1} m}{(n-2) ! \alpha^{n-2}} \int_{T_{0}}^{t} 1 / r_{1}(x) \int_{T_{0}}^{x}(x-s)^{n-2}|a(s)| d s d x \\
& +\frac{1}{(n-2) ! \alpha^{n-2}} \int_{T_{0}}^{t} 1 / r_{1}(x) \int_{T_{0}}^{x}(x-s)^{n-2}|b(s)| d s d x .
\end{aligned}
$$

Now there exists a constant $D_{i}>0$ such that for each $i$

$$
\left|\frac{r_{i+1}\left(T_{0}\right) z_{i} Y\left(T_{0}\right)}{\alpha^{i} \imath !} \int_{T_{0}}^{t} \frac{\left(x-T_{0}\right)^{i-2}}{r_{1}(x)} d x\right|
$$


$\leq\left|\frac{r_{i+1}\left(T_{0}\right) z_{i} y\left(T_{0}\right)}{\alpha^{i} 1 !}\right| D_{i} \int^{t} \frac{\left(x-T_{0}\right)^{i-2}}{x^{n-\beta}} d x$

$\leq\left|\frac{r_{i+1}\left(T_{0}\right) z_{i} Y\left(T_{0}\right)}{\alpha^{i} l !}\right| D_{i} \int_{T_{0}}^{t} \frac{1}{x^{n-i+2-\beta}} d x$

$\leq\left|\frac{r_{i+1}\left(T_{0}\right) z_{i} Y\left(T_{0}\right)}{\alpha^{i} 1 !}\right| D_{1} \cdot \frac{1}{n-i+1-\beta} \quad\left[\frac{1}{t^{n-i+1-\beta}}+\frac{1}{T_{0}^{n-i+1-\beta}}\right]$

$<\frac{\mathrm{d}}{\mathrm{n}}$

in view of (17), conveniently large enough choice of $T_{0}$ ) and the fact that $B<1$.

Similarly, as it was shown in the later part of inequality (14) (by changing the order of integration) it is easily shown that a large choice of $\mathrm{T}_{0}$ results in

$\left|\frac{c_{1} m}{(n-2) ! \alpha^{n-2}} \int_{T_{0}}^{t} 1 / x_{1}(x) \int_{T_{0}}^{x}(x-s)^{n-2}\right| a(s)|d s d x| \leq \frac{d}{n}$

and

$\left|\frac{1}{(n-2) ! \alpha^{n-2}} \int_{T_{0}}^{t} 1 / x_{1}(x) \int_{T_{0}}^{x}(x-s)^{n-2}\right| b(s)|d s d x| \leq \frac{d}{n}$.

From (21), (22), (23) and (24) we get

$|y(t)| \leq\left(\frac{d}{n}+\frac{d}{n}+\cdots-\frac{d}{n}\right)=d$

From (25) we see that if we choose a large enough $T_{0}$, then for all $t \geq T_{0}$ ' $|y(t)| \leq d$. But this contradicts (16) for any positive $d$. The proof is now complete.

EXAMPLE (3.1). The equation

$$
\begin{aligned}
\left(t\left(e^{t} y^{\prime}(t)\right)^{\prime}\right)^{\prime} & +e^{-t-2 \pi} y(t-\pi)=2 e^{-t} t \sin t+e^{-t} \sin t \\
& +4 t e^{-t} \cos t-3 e^{-t} \cos t-e^{-3 t} \sin t,
\end{aligned}
$$


$t>\pi$ has $y=e^{-2 t}$ sin $t$ as an oscillatory solution approaching zero. All conditions of Theorem 3.1 are satisfied. Hence all oscillatory solutions of (26) vanish at $\infty$.

our next theorem leads to a necessary and sufficient criteria for all oscillatory solutions of equation (1) to vanish at $\infty$.

THEOREM (3.2). Suppose $a(t)>0, \frac{1}{r_{1}(t)}=O\left(\frac{1}{t^{n-\beta}}\right)$, for $0 \leq \beta<1$ and $\int^{\infty} a(t) d t<\infty$. Further suppose that $b(t) / a(t)$ approaches $a$ finite limit as $t \rightarrow \infty$. Then a necessary and sufficient condition for all oscillatory solution of (1) to approach zero is

$$
\lim _{t \rightarrow \infty} \frac{|b(t)|}{a(t)}=0 \text {. }
$$

PROOF. (SUFFICIENCY). Suppose that $\frac{b(t)}{a(t)} \rightarrow 0$ as $t \rightarrow \infty$. Since $\int^{\infty} a(t) d t<\infty$, we have $\int^{\infty}|b(t)| d t<\infty$. By Theorem 3.1 all oscillatory solutions approach zero.

(NECESSITY). Let $y(t)$ be an oscillatory solution of (1).

Dividing (1) by $a(t)$ we have

$\frac{1}{a(t)}\left(r_{n-1}\left(r_{n-2}\left(--\left(r_{1} Y^{\prime}(t)\right)^{\prime}\right)^{\prime}\right)^{\prime}--\right)^{\prime}+h(y(g(t)))=\frac{b(t)}{a(t)}$

Now $y(t) \rightarrow 0$ as $t \rightarrow \infty$. Suppose to the contrary that

$$
\lim _{t \rightarrow \infty} \frac{\mid b(t)}{a(t)} \mid \geq \lambda>0 \text {. }
$$

Since $h(y(g(t))) \rightarrow 0,(28)$ from (29) reveals that there exists a large $T$ such that for $t \geq T, z_{n-1} y(t)>0$. But then $y(t)$ is nonoscillatory, a contradiction. The proof is now complete.

EXAMPLE (3.2). Consider the equation

$$
\left(t^{2} y^{\prime}(t)\right)^{\prime}+\frac{1}{t^{2}} y(t)=\frac{5}{t^{3}}\left(\sin \left(l_{n} t\right)-\cos \left(l_{n} t\right)\right)+\frac{\sin \left(l_{n} t\right)}{t^{5}}
$$

Here all conditions of Theorem 3.2 are satisfied. Hence all oscillatory solutions approach zero. In fact $y(t)=\sin \left(l_{n} t\right) / t^{3}$ is an oscillatory 
solution of $(30)$.

The necessity part of Theorem 3.2 leads us to the following theorem. THEOREM (3.3). Suppose $\frac{1}{r_{1}(t)}=O\left(1 / t^{n-\beta}\right)$ for some $\beta$ such that $0 \leq \beta<1$. Further suppose that $\int^{\infty} a(t) d t<\infty, a>0$ and $b(t) / a(t)$ is bounded. Then all oscillatory solution of equation (1) approach zero as $t \rightarrow \infty$.

PROOF. Since $\int^{\infty} a(t)<\infty$, boundedness of $b(t) / a(t)$ implies $\int^{\infty}|b(t)| d t<\infty$. Conditions of Theorem 3.1 hold. The proof is complete. our next Theorem gives conditions when oscillatory solutions do not approach limits.

THEOREM (3.4). Suppose $a(t)>0$ and $\lim _{t \rightarrow \infty} \inf |b(t)| / a(t)>0$.

Let $y(t)$ be an oscillatory solution of equation (1). Then $\lim \sup |y(t)|>0$. $t \rightarrow \infty$

PROOF. Suppose to the contrary that $y(t) \rightarrow 0$ as $t \rightarrow \infty$. Then $h(y(g(t))) \rightarrow 0$. From equation (I) $\frac{1}{a(t)} \mid\left(r_{n-1}\left(r_{n-2}\left(--\left(r_{2}(t)\left(r_{1}(t) y^{\prime}(t)\right)\right)^{\prime} \cdot--\right) \cdot|+| h(y(g(t)))|\geq| b(t) \mid / a(t)\right.\right.$. This shows that $z_{n-1} y(t)$ is eventually positive contradicting the fact that $y(t)$ is oscillatory.

REMARK. It is to be noted that the conditions

$$
\frac{1}{r_{1}(t)}=O\left(\frac{1}{t^{n-\beta}}\right), \int^{\infty} a(t) d t<\infty \text { and } \int^{\infty}|b(t)| d t<\infty
$$

are not needed here.

EXAMPLE (3.3). All oscillatory solutions of the equation

$$
y^{\prime \prime}(t)+y(t-2 \pi)=2
$$

satisfy $\lim _{t \rightarrow \infty} \sup _{y}|y(t)|>0$ since this equation satisfies all conditions of Theorem 3.4. $y(t)=2+2 \cos (t)$ is one such solution. 
Next theorem gives nonoscillation criterion.

THEOREM (3.5). Suppose $a(t)>0, \int^{\infty} a(t) d t<\infty$ and $\frac{1}{r_{1}(t)}=O\left(\frac{1}{t^{n-\beta}}\right), 0 \leq \beta<1$. Further suppose that $\underset{t \rightarrow \infty}{\lim \inf |b(t)| / a(t)>0}$ and $b(t) / a(t)$ is bounded. Then all solutions of equation (1) are nonoscillatory.

PROOF. Suppose to the contrary that $y(t)$ is an oscillatory solution of (1). Since all conditions of Theorem 3.3 are satisfied, $y(t) \rightarrow 0$ as $t \rightarrow \infty$. Thus $h(y(g(t))) \rightarrow 0$. From equation (1)

$\frac{1}{a(t)}\left|\left(r_{n-1}\left(r_{n-2}(t)\left(--\left(r_{1}(t) y^{\prime}(t)\right)\right)^{\prime}\right)^{\prime} \cdot---\right) \cdot\right| \geq|b(t)| / a(t)-|h(y(g(t)))|$.

(32) suggests that $z_{n-1} y(t)>0$ eventually, contradicting the fact that $y(t)$ is oscillatory. The proof is now complete.

EXAMPLE (3.5). The equation

$$
\left(\frac{1}{2} t^{2} y^{\prime}(t)\right)^{\prime}+\frac{1}{t^{2}} y(t)=\frac{1}{t^{2}}+\frac{1}{t^{4}}
$$

satisfies all conditions of this theorem. $y(t)=1 / t^{2}$ is a nonoscillatory solution of (33).

THEOREM (3.6). Suppose. $\int^{\infty}|a(t)| d t<\infty$ and $\left|\int^{\infty} b(t) d t\right|=\infty$. Then all oscillatory solutions of (1) are unbounded.

PROOF. Suppose to the contrary that some oscillatory solution $y(t)$ satisfies $|y(t)| \leq c_{0}$ for some $c_{0}>0$. From equation (1) on integration for $t \geq T$.

$\left|r_{n-1}(t) z_{n-2} y(t)\right|+\left|r_{n-1}(T) z_{n-2} y(T)\right|+c_{0} m \int_{T}^{t}|a(s)| d s \geq\left|\int_{T}^{t} b(s) d s\right|$.

(34) yields that $z_{n-2} y(t)$ assumes a constant sign eventually, contradicting that $y(t)$ is oscillatory. The proof is now complete. The following example shows that under the conditions of Theorem 3.6, it is possible to have bounded nonoscillatory solutions. 
EXAMPLE (3.6). The equation

$$
\left(t^{5 / 2}\left(t y^{\prime}(t)^{\prime}\right)^{\prime}+\frac{1}{t^{2}} y(t)=\frac{1}{t^{3}}+\frac{1}{2 \sqrt{t}}\right.
$$

satisfies all conditions of Theorem 3.6. It has $y(t)=1 / t$ as a bounded nonoscillatory solution.

THEOREM (3.7). Suppose $\int^{\infty}|a(t)| d t<\infty$ and $\int^{\infty} b(t) d t= \pm \infty$. Further suppose that $r_{i}(t)$ is bounded $i=1,2,---, n-1$. Then all solutions of equation (1) are unbounded.

PROOF. Due to Theorem 3.6 we only need to prove it for a nonoscillatory solution. Let $y(t)$ be nonoscillatory and bounded. From inequality (34) in the proof of Theorem 3.6 it follows that $\left|z_{n-2} y(t)\right| \rightarrow \infty$ as $t \rightarrow \infty$. Since $z_{n-2} Y=\left(r_{n-2} z_{n-3} Y(t)\right)$, and $r_{n-2}$ is bounded, we have $z_{n-3} Y(t) \rightarrow \pm \infty$. Proceeding this way we find that $y^{\prime}(t) \rightarrow \pm \infty$ forcing $y(t) \rightarrow \pm \infty$. The proof is now complete by contradiction.

FINAI REMARK. Theorem 3.1 improves our main result in [9] (c.f [11]) where it was shown that oscillatory solutions of

$$
\left(r(t) y^{\prime}(t)\right)^{(n-1)}+a(t) h(y(g(t)))=f(t)
$$

approach zero subject to:

$$
\int^{\infty}|a(t)| t^{n-2} d t<\infty, \quad \int^{\infty}|f(t)| t^{n-2} d t<\infty
$$

and

$$
\frac{1}{r(t)}=O\left(\frac{.1}{t^{n-\beta}}\right), \quad 0 \leq \beta<1
$$

The restriction on $r(t)$ cannot be weakened i.e. $B$ cannot be greater than or equal to 1 as the following example shows.

- EXAMPLE (3.7). The equation

$$
\begin{gathered}
\left(t^{2} y^{\prime}(t)\right) \cdots+\frac{1}{t\left(l_{n} t\right)^{2}} y(t)=\frac{\cos \left(l_{n}\left(l_{n} t\right)\right)}{t\left(l_{n} t\right)^{3}}+\frac{3 \sin \left(l_{n}\left(l_{n} t\right)\right)}{t\left(l_{n} t\right)^{3}}-\frac{\cos \left(l_{n}\left(l_{n} t\right)\right)}{t\left(l_{n} t\right)^{2}} \\
t>0
\end{gathered}
$$


has $y=\sin \left(l_{n}\left(l_{n} t\right)\right)$ as an oscillatory solution which does not have a limit at $\infty$. Only the condition on $r(t)$ is violated. We see that for $n=3$ \[ \frac{1}{r(t)}=\frac{1}{t^{n-1}} \text { so that } \beta=1 \]
even though $\int^{\infty} \frac{1}{r(t)} d t<\infty$

\section{REFERENCES}

1. Chen, L. S. On the Oscillation and Asymptotic Properties for General Nonlinear Differential Equations, Atti. Accad. Naz. Lincei. Rend. Cl. Sci. Fis Mat Natur 51 (1976) pp. 211-216.

2. Graef, J. R. Oscillation, Nonoscillation and Growth of Solutions of Nonlinear Functional Differential Equations of Arbitrary Order, J. Math. Anal. Appl. 60 (1977) 398-409.

3. Hammett, M. E. Nonoscillation Properties of a Nonlinear Differential Equation, Proc. Amer. Math. Soc. 30 (1971) 92-96.

4. Kartsatos, A. G. Oscillation and Existence of Unique Positive Solutions for Nonlinear nth Order Equations with Forcing Term, Hiroshima Math. J. $\underline{6}$ (1976) 1-6.

5. Kusano, T. and H. Onose. Asymptotic Behavior of Nonoscillatory Solutions of Second Order Functional Differential Equations, Bull. Austral. Math. Soc. 13 (1975) 291-299.

6. Kusano, T. and H. Onose. Nonoscillation Theorems for Differential Equations with Deviating Argument, Pacific J. Math. 62 (1976) 185-192.

7. Singh, B. and R. S. Dahiya. On Oscillation of Second Order Retarded Equations, J. Math. Anal. Appl. 47 (1974) 504-512.

8. Singh, B. Asymptotically Vanishing Oscillatory Trajectories in Second order Retarded Equations, SIAM J. Math. Anal. 7 (1976) 37-44.

9. Singh, B. A Correction to "Forced Oscillations in General Ordinary Differential Equations with Deviating Arguments HMJ 6 (1976)", Hiroshima Math. J. (to appear).

10. Singh, B. Nonoscillation of Forced Fourth Order Retarded Equations, SIAM J. Appl. Math. 28 (1975) 265-269.

11. Singh, B. Asymptotic Nature of Nonoscillatory Solutions of nth order Retarded Differential Equations, SIAM J. Math. Anal. 6 (1975) 784-795. 
12. Singh, B. General Functional Differential Equations and Their Asymptotic Oscillatory Behavior, Yokahama Math. J. 24 (1976) 125-132.

13. Singh, B. Forced Oscillations in General Ordinary Differential Equations with Deviating Arguments, Hiroshima Math. J. $\underline{6}$ (1976) 7-14. 


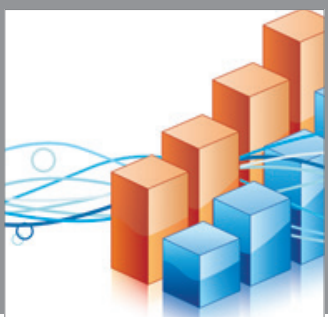

Advances in

Operations Research

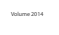

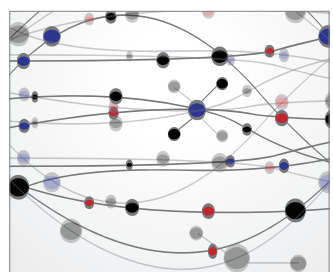

\section{The Scientific} World Journal
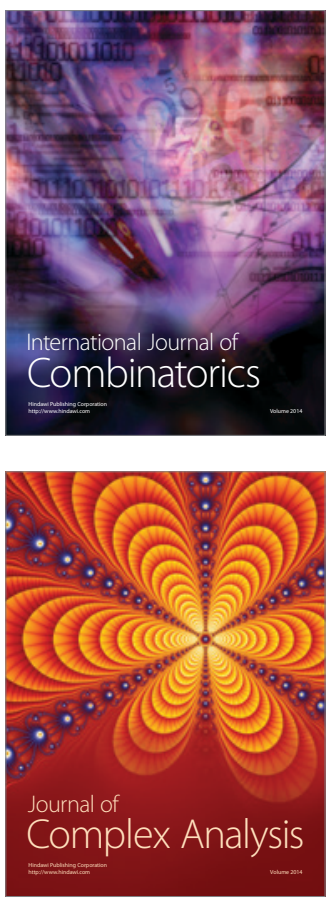

International Journal of

Mathematics and

Mathematical

Sciences
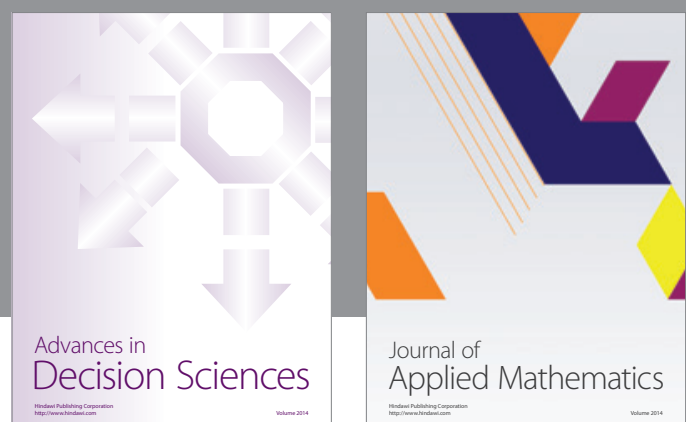

Journal of

Applied Mathematics
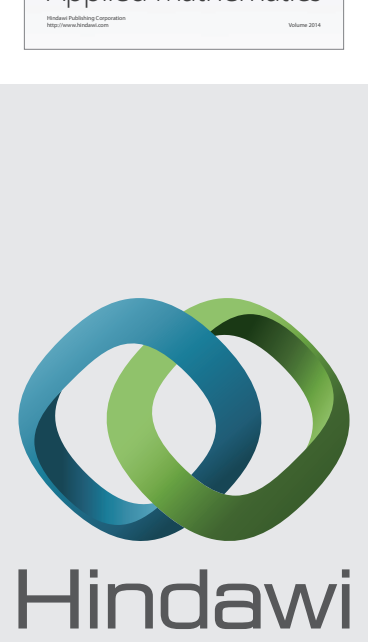

Submit your manuscripts at http://www.hindawi.com
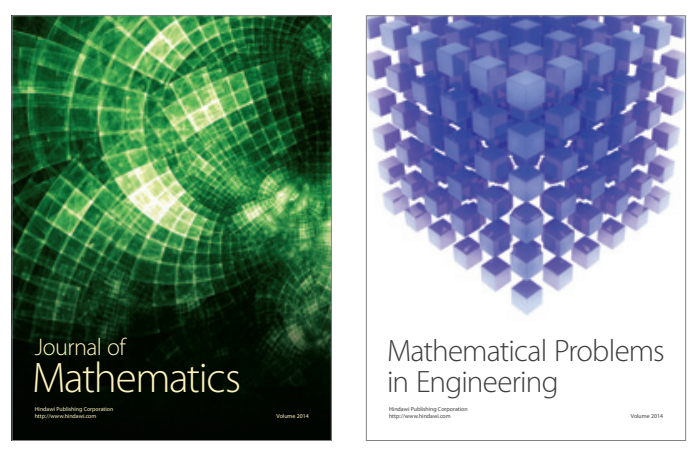

Mathematical Problems in Engineering
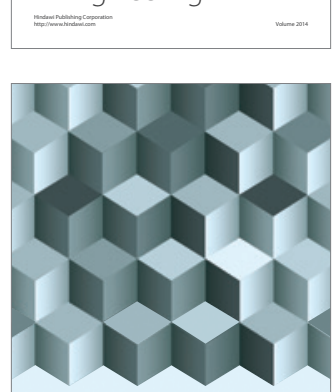

Journal of

Function Spaces
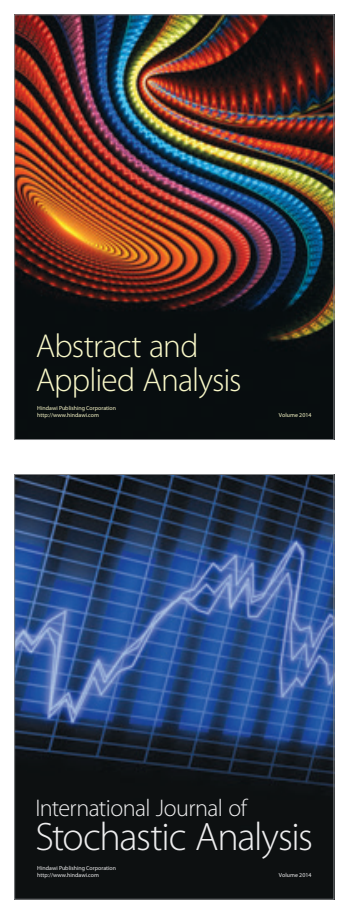

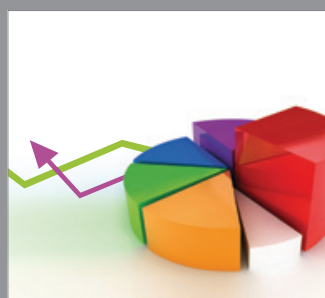

ournal of

Probability and Statistics

Promensencen
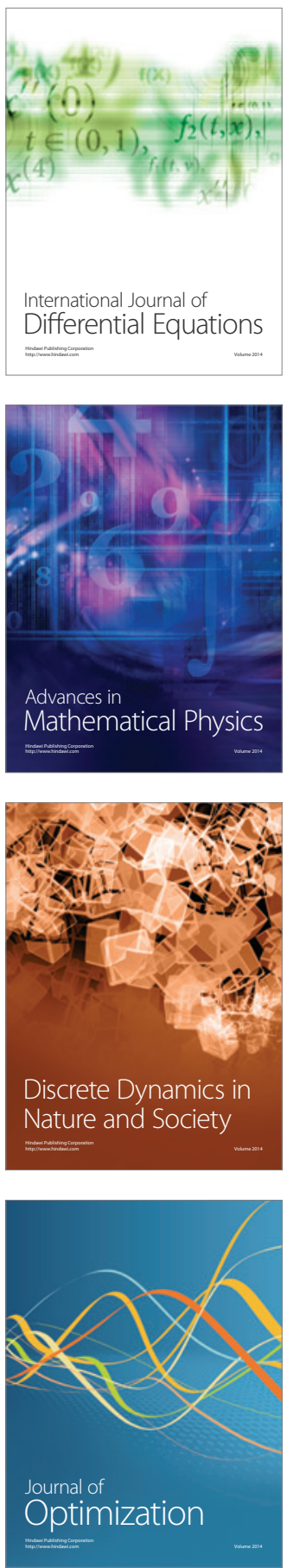